\title{
Dimethyl sulfoxide induces chemotherapeutic resistance in the treatment of testicular embryonal carcinomas
}

\author{
HIROKO KITA $^{1,2}$, KEISEI OKAMOTO ${ }^{2}$, RYOJI KUSHIMA ${ }^{1}$, AKIHIRO KAWAUCHI ${ }^{2}$ and TOKUHIRO CHANO $^{1}$ \\ Departments of ${ }^{1}$ Clinical Laboratory Medicine and ${ }^{2}$ Urology, Shiga University of Medical Science, \\ Otsu, Shiga 520-2192, Japan
}

Received August 8, 2014; Accepted April 21, 2015

DOI: 10.3892/ol.2015.3306

\begin{abstract}
Dimethyl sulfoxide (DMSO) is an amphipathic molecule that is used as a solvent in biological studies and as a vehicle for drug therapy. The present study was designed to evaluate the potential effects of DMSO as a solvent in the treatment of testicular embryonal carcinomas (ECs). DMSO was applied to two human EC cell lines (NEC8 and NEC14), with the treated cells evaluated in relation to cisplatin (CDDP) resistance, differentiation (using Vimentin, Fibronectin, TRA-1-60, and SSEA-1 and -3 as markers) and stemness (denoted by expression of SOX2 and OCT3/4). Furthermore, DNA methyltransferase (DNMT-1, -3A and -3L) expression and methylation status were analyzed. DMSO induced resistance to $\mathrm{CDDP}$, aberrant differentiation and reduction of stemness-related markers in each of the EC cell lines. The expression levels of DNMT-3L and -3A were reduced in response to DMSO, while this treatment also affected DNA methylation. The data demonstrated that DMSO perturbed differentiation, reduced stemness and induced resistance to CDDP in human EC cells. Therefore, DMSO could reduce drug efficacy against EC cells and its use should be carefully managed in the clinical application of chemotherapy.
\end{abstract}

\section{Introduction}

Dimethyl sulfoxide (DMSO) is a solvent with amphiphathic properties that is capable of dissolving a wide range of substances, and is used as a solvent in biological studies and as a vehicle for drug therapy (1). cis-Diamminedichloroplatinum (II) (cisplatin; CDDP) is widely used in the treatment of various types of cancer. CDDP-containing chemotherapy regimens have been shown

Correspondence to: Professor Tokuhiro Chano, Department of Clinical Laboratory Medicine, Shiga University of Medical Science, Tsukinowa-cho, Seta, Otsu-shi, Shiga 520-2192, Japan

E-mail: chano@belle.shiga-med.ac.jp

Key words: embryonal carcinoma, dimethyl sulfoxide, cisplatin, differentiation, methylation to be effective in the clinical management of testicular embryonal carcinomas (ECs) (2). CDDP is not soluble in water; accordingly, DMSO is often used as a solvent for it or for other combined agents in biological experiments and in the clinical setting.

Testicular germ cell tumors (TGCTs) are the most frequently occurring malignancy in males aged 15-35 years. While TGCTs are classified into two major histological subgroups, namely seminomas and non-seminomatous germ cell tumors (NSGCTs), they are suggested to all arise from the same precursor (3). The clinical outcome of patients with NSGCTs tends to be worse for those with seminomas. NSGCTs exhibit embryonal and extraembryonal differentiation patterns, including primitive zygotic (EC), embryonal-like somatic differentiated (teratoma) and extraembryonally differentiated (choriocarcinoma and yolk sac tumor) phenotypes (4). The majority of NSGCTs contain ECs, with these displaying similarities to embryonic stem (ES) cells and being able to differentiate into essentially any tissue (5), including yolk sac tumors, choriocarcinoma and teratoma. Due to advances in combination chemotherapy, the 10 -year disease-specific survival rate in patients with metastatic NSGCTs is $88 \%$ (6). However, $30 \%$ of patients with stage I NSGCTs relapse during active surveillance (7-9). Chemotherapy containing CDDP has been introduced as an adjuvant treatment option for micrometastatic disease, thereby reducing the risk of relapse to $2 \%$ (10). The percentage of EC component in the primary tumor is an important predictive factor for occult metastasis in stage I NSGCTs (7,11-13), and adjuvant chemotherapy should be performed in such a way that resistance is minimized (14). Therefore, EC component control without induction of chemotherapeutic resistance is crucial for the appropriate clinical management of NSGCTs when considering the fact that ECs are a putative cancer stem component of NSGCT (15).

DMSO has been shown to induce differentiation in EC and ES cells, and to affect other cellular functions, including progression through the cell cycle and apoptosis (1). If DMSO is utilized to solubilize chemotherapeutic agents, such as CDDP, in the clinical management of NSGCTs, it can affect the character of EC components and reduce drug efficacy. Thus, the present study was designed to clarify the potential effects of DMSO on EC cells and the associated responses to CDDP. 


\section{Materials and methods}

Cell lines and culture conditions. Two EC cell lines, namely NEC8 and NEC14 (Riken Cell Bank, Tsukuba, Japan), were used in the present study. The cell lines were maintained in RPMI 1640 (Life technologies, Tokyo, Japan) containing $10 \%$ fetal bovine serum (FBS; GE healthcare Life Sciences, Chalfont, UK) supplemented with penicillin $(100 \mathrm{U} / \mathrm{ml})$, streptomycin $(100 \mathrm{U} / \mathrm{ml})$ and glutamine $(300 \mathrm{mg} / \mathrm{l})$ in a humidified atmosphere of $5 \% \mathrm{CO}_{2}$ on dishes coated with type I collagen. The cells were passaged when they reached $80 \%$ confluence. Experimental procedures using the cell lines were performed within 6 months of receipt from the cell bank.

DMSO treatment. The NEC8 and NEC14 cells were exposed to DMSO (Sigma-Aldrich, St. Louis, MO, USA) for $72 \mathrm{~h}$ after plating and the effects of DMSO were examined and compared with untreated control cells. DMSO was diluted in the culture medium, with resulting final concentrations of $0.2,0.4,0.6$, 0.8 and $1.0 \%(\mathrm{v} / \mathrm{v})$, respectively.

Cell counting kit-8 (CCK-8) assay. The NEC8 and NEC14 cells were seeded at $3 \times 10^{3}$ cells/well in 96-well plates coated with type I collagen containing RPMI 1640 with $10 \%$ FBS, and incubated at $37^{\circ} \mathrm{C}$. The next day, DMSO was applied at the various aforementioned concentrations. After $72 \mathrm{~h}$ of DMSO treatment, CDDP was applied at a final concentration of $0,2.5,5,7.5,10,15$, and $20 \mu \mathrm{M}$, respectively. The cells were incubated in presence or absence of CDDP for 2 days. Cell viability was subsequently measured by WST- 8 assay using CCK-8 (Dojindo Molecular Technologies Inc., Kumamoto, Japan). WST-8 reagent solution was added to each well, and the cell plates were incubated for $3 \mathrm{~h}$. Next, the absorbance of each well was measured using an Infinite M200 microplate reader (Tecan, Männedorf, Switzerland) at a wavelength of $450 \mathrm{~nm}$.

Western blot analysis. The cells were lysed in Laemmli-sodium dodecyl sulfate (SDS) buffer, and the lysate was sonicated and then boiled for $5 \mathrm{~min}$. Protein lysate from U-2 OS/DOXO35 (16) cells was used as a positive control for MDR-1 expression. Samples containing equal amounts of protein were separated via SDS-polyacrylamide gel electrophoresis on 6-10\% gels prior to transfer to polyvinylidine fluoride membranes (Millipore, Billerica, MA, USA). The membranes were immunoblotted with the following primary antibodies: mouse monoclonal anti-MDR-1 (1:100, JSB-1; Abcam, Cambridge, UK) and mouse monoclonal anti-MRP-1 (1:100; MRPm6; Enzo Life Sciences, Farmingdale, NY, USA), which are drug efflux pumps; rabbit monoclonal anti-Vimentin $(1: 1,000$; D21H3; Cell Signaling Technology Inc., Danvers, MA, USA), mouse monoclonal anti-Fibronectin (1:500; NCL-FIB, \#568; Leica Microsystems Inc., Buffalo Grove, IL, USA) and mouse monoclonal anti-TRA-1-60 (1:500; TRA-1-60; eBioscience Inc., San Diego, CA, USA), as markers of differentiation; rabbit polyclonal anti-SOX2 (1:500, \#2748; Cell Signaling Technology Inc.); and goat polyclonal anti-OCT3/4 (1:250; C-20; Santa Cruz Biotechnology Inc., Dallas, TX, USA), as a marker of stemness. Mouse monoclonal anti-DNMT1
(1:500; 60B1220-1; Abcam), rabbit polyclonal anti-DNMT3A (1:1,000: 64B1446; Novus Biologicals, LLC, Littleton, CO, USA) and mouse monoclonal anti-DNMT3L (1:1,000; Novus Biologicals, LLC) (17) were used for DNMT family screening. Anti- $\alpha$ tubulin (1:2,000; DM1A; Sigma-Aldrich) was utilized to provide a loading control.

Fluorescence immunocytochemistry. The NEC8 and NEC14 cells were cultured on type I collagen-coated LabTek ${ }^{\mathrm{TM}}$ chamber slides (BD Biosciences, Franklin Lakes, NJ, USA) to $50 \%$ confluence, fixed with $2 \%$ buffered-formaldehyde and $70 \%$ ethanol, and then permeabilized with $0.1 \%$ Triton X-100. The primary mouse anti-TRA-1-60 (1:200; TRA1-60; eBioscience), mouse anti-SSEA-1 (1:200; MC-480, Abcam), rat anti-SSEA-3 (1:50; MC-631, R\&D systems, Minneapolis, MN, USA) and rabbit anti-DNMT3L antibodies (17) were incubated overnight at $4^{\circ} \mathrm{C}$. Appropriate secondary antibodies labeled with Alexa 488 (Life Technologies) were then applied, followed by washing with phosphate-buffered saline (PBS) to remove excess fluorescent dye, and mounting with glycerol. The specimens were observed and images were captured under identical conditions using a fluorescence microscope fitted with a charge-coupled device camera (DMI4000 B; Leica Microsystems Inc.).

DNA extraction. The NEC8 and NEC14 cells were washed with PBS and suspended in lysis buffer $[10 \mathrm{mM}$ Tris-HCl and 50 mM EDTA (pH 8.0), $10 \mathrm{mM} \mathrm{NaCl}, 2 \% N$-lauryl sarcosine and $200 \mu \mathrm{g} / \mathrm{ml}$ proteinase $\mathrm{K}]$. The mixture was incubated for $20 \mathrm{~h}$ at $55^{\circ} \mathrm{C}$, followed by phenol chloroform extraction and ethanol precipitation. DNA from cell lines was extracted using QIAamp DNA Blood Mini kits (Qiagen Inc., Valencia, CA, USA) and human genomic DNA from peripheral blood lymphocytes was obtained from Takara Bio Inc. (Otsu, Shiga, Japan).

Treatment of DNA with sodium bisulfite. The method described by Clark et al (18) was used to perform bisulfite treatment, with alterations used as detailed by Frevel et al (19). The bisulfite reaction, under mineral oil, was performed at $55^{\circ} \mathrm{C}$ for $16 \mathrm{~h}$ in a $525-\mu 1$ total volume containing $2.4 \mathrm{M}$ sodium bisulfite (Sigma-Aldrich) and $123 \mathrm{mM}$ hydroquinone (Sigma-Aldrich). Reactions were desalted using a QIAEX II Gel Extraction kit (Qiagen Inc.). DNA was eluted in $50 \mu 1$ $\mathrm{H}_{2} \mathrm{O}$, incubated with $5 \mu \mathrm{l}$ of $3 \mathrm{M} \mathrm{NaOH}$ for $15 \mathrm{~min}$ at $37^{\circ} \mathrm{C}$, neutralized with ammonium acetate (final concentration of $3 \mathrm{M}$ ) and ethanol precipitated. The bisulfite-treated DNA was then resuspended in $25 \mu 1 \mathrm{H}_{2} \mathrm{O}$ and stored at $-20^{\circ} \mathrm{C}$.

Combined bisulfite restriction analysis (COBRA) for LINE1. COBRA was conducted as described previously (20). Methylation of the LINE1 promoter was investigated as follows: Polymerase chain reaction (PCR) amplification was performed in 25- $\mu 1$ volumes using Ex Taq buffer (Takara Bio Inc.) under the following conditions: $2 \mathrm{mM} \mathrm{MgCl}_{2}$, $200 \mathrm{mM}$ of each deoxynucleotide triphosphate, $0.8 \mathrm{mM}$ final concentration of each primer and 0.6 units of Ex Taq buffer (Takara Bio Inc.). The primer sequences associated with the LINE1 promoter region were: 5'-TTGAGTTGTGGT GGGTTTTATTTAG-3' (496-520, X58075) and 5'-TCATCT 
A

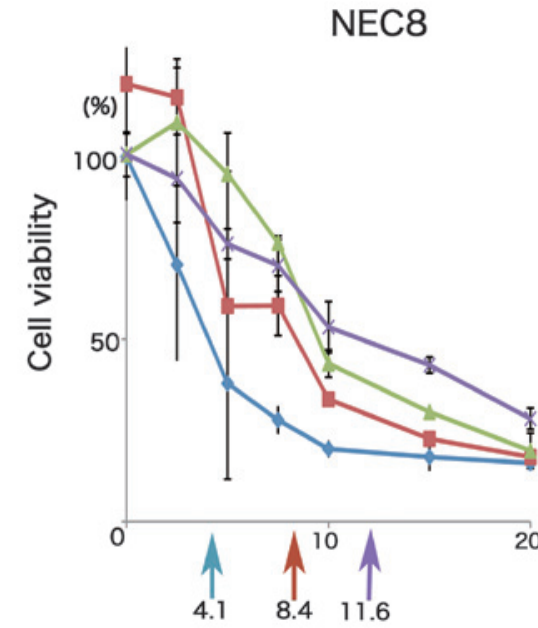

B
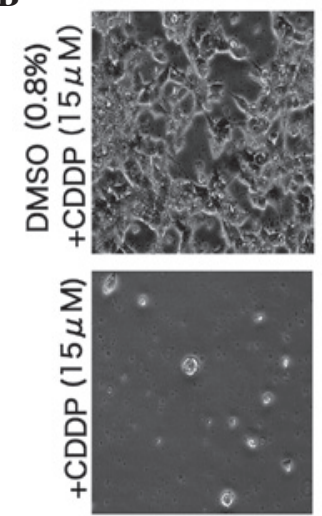

NEC8
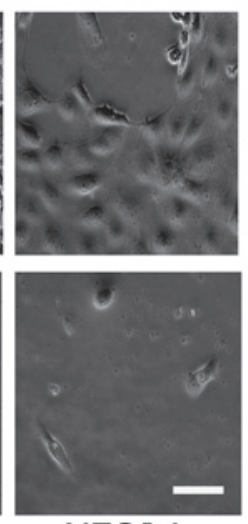

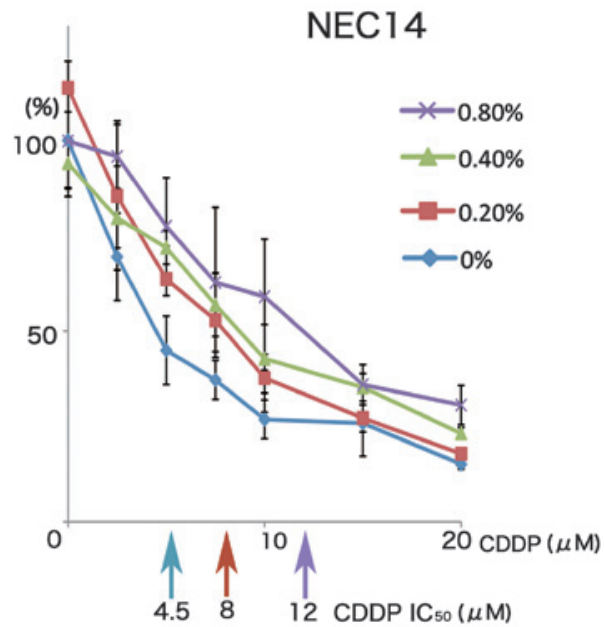

C

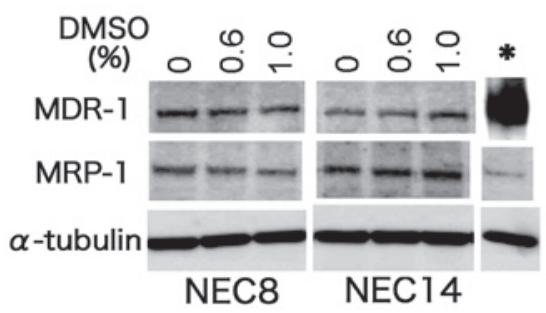

Figure 1. DMSO enhances resistance to CDDP without induction of MDR-1 or MRP-1 in human EC cells. (A) Viability of human EC cells after 48 h of CDDP exposure together with DMSO treatment. Two EC cell lines, NEC8 and NEC14, were treated with DMSO at the indicated concentrations for $72 \mathrm{~h}$, and various doses of CDDP were additionally applied for $48 \mathrm{~h}$. The effects on cell growth were determined by use of the WST- 8 assay ( $\mathrm{n}=3$; vertical bars, standard deviation). $\mathrm{IC}_{50}$ values were indicated by each colored-arrow and value. DMSO decreased the cytotoxicity of CDDP in a dose-dependent manner; $0.8 \%$ (v/v) DMSO induced $\sim 3$-fold more resistance to CDDP than 0\% (v/v) DMSO in each cell line. (B) Micrographs of NEC8 and NEC14 cells cultured in medium containing $15 \mathrm{mM}$ CDDP with/without $0.8 \%$ (v/v) DMSO. The NEC8 and NEC14 cells retained their morphology at high doses of CDDP with DMSO, with exposure to CDDP alone leading to a significant reduction in cell numbers. Scale bar, $100 \mu \mathrm{m}$. (C) Western blot analysis of the major drug efflux pumps, MDR-1 and MRP-1. No induction of either pump was observed in response to DMSO treatment in the NEC8 and NEC14 cells. Cell lysate $(*)$ of U-2OS/DOXO35 was used as the positive control for MDR-1. DMSO, dimethyl sulfoxide; CDDP, cisplatin; EC, embryonal carcinoma; $\mathrm{IC}_{50}$, half maximal inhibitory concentration.

CACTAAAAAATACCAAACA-3' (108-132, X58075). The PCR cycling conditions were $95^{\circ} \mathrm{C}$ for $30 \mathrm{sec}, 50^{\circ} \mathrm{C}$ for $30 \mathrm{sec}$ and $72^{\circ} \mathrm{C}$ for $30 \mathrm{sec}$ for 35 cycles. The final PCR product was digested with the HinfI restriction enzyme (Takara Bio Inc.). The digested PCR products were separated by electrophoresis on $6 \%$ polyacrylamide gels. In a COBRA analysis, the lower digested bands represent methylated repetitive elements and the upper undigested band represents unmethylated repetitive elements or repetitive elements in which the restriction site has been mutated. Following gel electrophoresis and ethidium bromide staining, the PCR bands were quantitated through densitometric analysis and the degree of methylation was thereby determined for LINE1 elements in the NEC8 and NEC14 cells.

Statistical analysis. A two-way factorial analysis of variance and multiple comparison tests accompanied by Scheffe's significance test were performed using StatView software for Windows, version 5.0 (SAS Institute, Cary, NC, USA). $\mathrm{P}<0.05$ was considered to indicate a statistically significant difference.

\section{Results}

DMSO enhances resistance to CDDP in EC cells without induction of drug efflux pumps. DMSO significantly induced the resistance to CDDP in the NEC8 and NEC14 cells. The CDDP half maximal inhibitory concentration $\left(\mathrm{IC}_{50}\right)$ values in the NEC8 cells varied from 4.1 to $11.6 \mu \mathrm{M}$ at 0 to $0.8 \%(\mathrm{v} / \mathrm{v})$ of DMSO exposure, conferring up to 3 -fold more resistance to CDDP than the no DMSO control ( $\mathrm{P}=0.0012$; Fig. 1A). Similarly, the NEC14 cells were $\sim 3$-fold more resistant to CDDP when exposed to $0.8 \%$ (v/v) DMSO ( $\mathrm{P}<0.001$; Fig. 1B). However, DMSO exposure did not result in the induction of the drug efflux pumps, MDR-1 and MRP-1, in either EC cell line (Fig. 1C). 
A

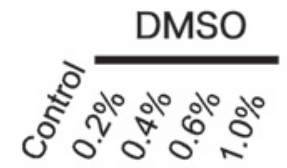

Vimentin

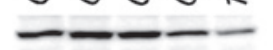

Fibronectin

TRA1-60

\section{SOX2}

ОСТ $3 / 4$

$\alpha$-tubulin

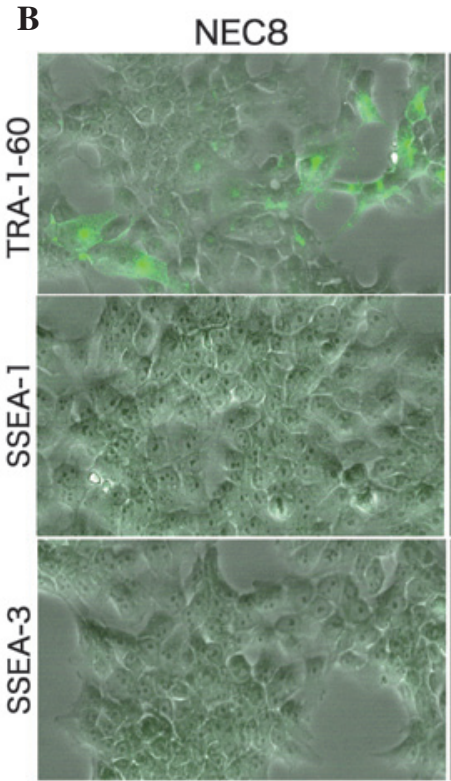

Control

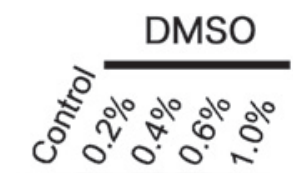

- - - - -
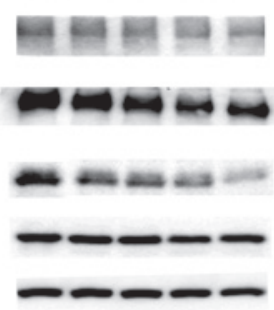

NEC14

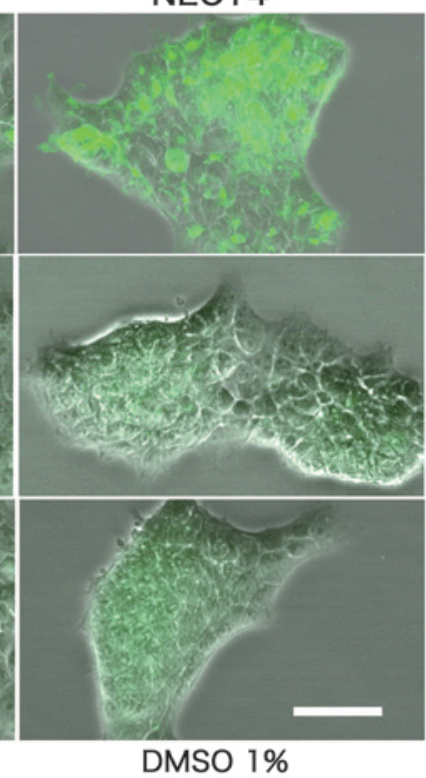

Figure 2. DMSO perturbs differentiation and reduces the stemness characteristics of human embryonic carcinoma cells. (A) Western blotting. Vimentin, Fibronectin and TRA-1-60 were used as markers of differentiation. SOX2 and OCT3/4 were used as stemness-related markers. DMSO dose-dependently induced the aberrant expression of Vimentin and TRA-1-60 in the NEC8 and NEC14 cells. DMSO also reduced SOX2 expression. $\alpha$-tubulin was used as a loading control. (B) Immunocytochemical evaluation of TRA-1-60, SSEA-1 and SSEA-3 in the NEC8 cells. DMSO significantly induced TRA-1-60 expression, but did not affect SSEA-1 and SSEA -3 expression. Scale bar, $100 \mu \mathrm{m}$. DMSO, dimethyl sulfoxide; CDDP, cisplatin.

DMSO perturbs differentiation and reduces stemness-related markers in EC cells. In order to detail the differentiation status of EC cells exposed to DMSO, the expression levels of Vimentin, Fibronectin, TRA-1-60 and SSEA-1 and -3 proteins were evaluated. DMSO dose-dependently induced TRA-1-60 expression in the NEC8 cells, but had no effect on this protein in the NEC14 cells. Vimentin protein expression was reduced dose-dependently by DMSO in each cell line (Fig. 2A-B). DMSO did not alter the expression of Fibronectin protein (Fig. 2A). SSEA-1 and -3 were subtly expressed in each EC cell line, with no differences in expression detected following DMSO exposure (Fig. 2A and B). Collectively, these data indicated that DMSO promoted aberrant differentiation in the human EC cells. To evaluate whether DMSO affects the stemness of EC cells, the present study analyzed several stemness-related markers in the

A
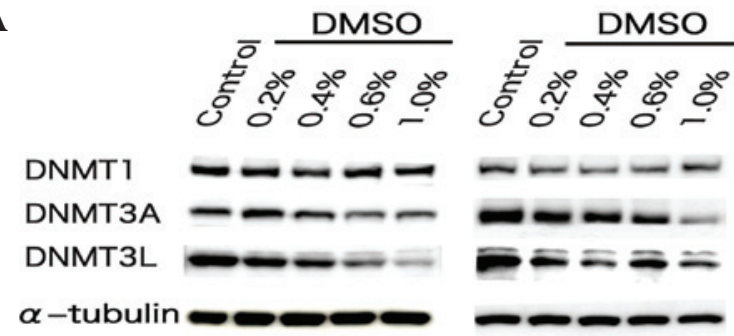

B

NEC8

NEC 14
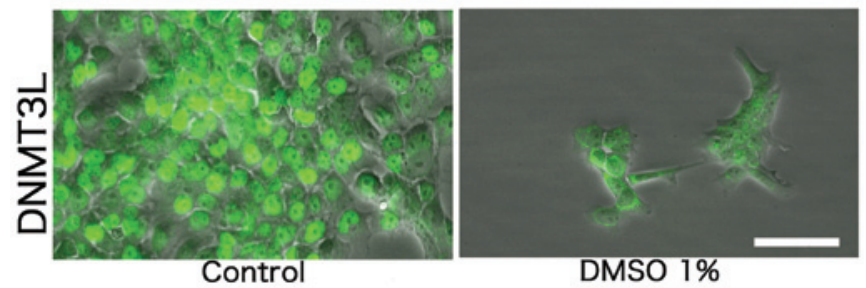

C
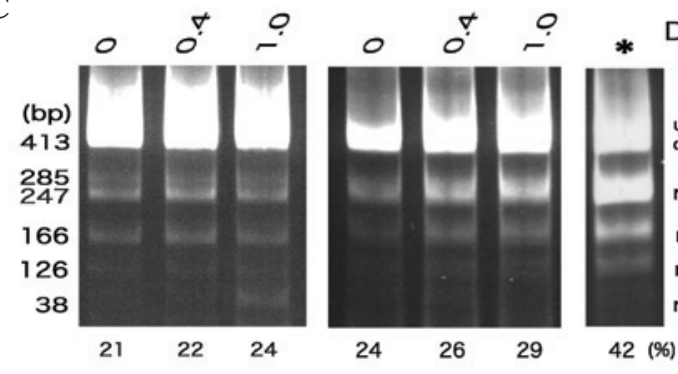

NEC8

Figure 3. DMSO reduces DNMT-3L and -3A expression, and increases DNA methylation levels in human embryonic carcinoma cells. (A) Western blot analysis for the DNMT family. DNMT-3A and -3L expression was dose-dependently reduced by DMSO in the NEC8 and NEC14 cells. The expression of DNMT1 showed little change between the control and DMSO-treated cells. (B) Immunocytochemical evaluation of DNMT3L in the NEC8 cells. DNMT3L was reduced and delocalized from the cell nuclei by DMSO exposure. Scale bar, $100 \mu \mathrm{m}$. (C) Methylation status of LINE1 DNA repetitive elements in the NEC8 and NEC14 cells. This COBRA assay indicated five possible digestion products of 285, 247, 166, 126 and $38 \mathrm{bp}$, signifying methylated repetitive elements. The upper undigested bands represent unmethylated repetitive elements or repetitive elements in which the restriction site has been mutated. The size of the undigested and digested PCR products is shown on the left. The reference mark (*) indicates a male PBL sample that was a highly methylated LINE1 control. The band intensities were quantitated through densitometric analysis, with the extent of methylation shown below each lane. DMSO, dimethyl sulfoxide; CDDP, cisplatin.

NEC8 and NEC14 cells. DMSO reduced SOX2 protein expression dose-dependently in the two cell types, although OCT3/4 protein expression was unchanged (Fig. 2A).

DMSO treatment reduces DNMT-3L and -3A expression, and up-regulates DNA methylation in EC cells. Testicular EC cells possess a strong tendency to maintain the entire genome in a demethylated state, distinguishing them from somatic cancer cells, which commonly display genomic hypermethylation $(21,22)$. In addition, DNMT-3L is a significant marker of human embryonic carcinoma (17). Therefore, the expression of several DNMT family members, as well as the methylated DNA status, was evaluated in the NEC8 and NEC14 cells following DMSO exposure. DMSO had no effect on DMNT1 
expression, but significantly reduced the expression of DNMT-3L and -3A (Fig. 3A). Moreover, DNMT3L was aberrantly delocalized from the cell nuclei by DMSO in the EC cells (Fig. 3B). While conducting COBRA of LINE1 repetitive elements, the two EC cell lines showed dose-dependent increases in the degree of LINE1 methylation after $72 \mathrm{~h}$ of treatment with DMSO (Fig. 3C).

\section{Discussion}

The majority of TGCTs are curable, even in the metastatic stages, as they are extremely sensitive to CDDP-based chemotherapy. However, certain NSGCT cases are refractory to any type of chemotherapy. EC is regarded as a stem cell component for NSGCT (15). EC cells may acquire resistance to chemotherapy in parallel with aberrant differentiation $(23,24)$. Thus, EC component control, without triggering chemotherapeutic resistance or perturbation of differentiation, is crucial for the effective clinical management of NSGCTs (15). The present study results indicated that DMSO can induce resistance to CDDP and can perturb the differentiation status of human EC cells. DMSO is frequently used as a solvent in biological studies and as a vehicle for drug therapies $(25,26)$. Even if DMSO is used as a vehicle for other drugs, when these agents are used in combination with CDDP, DMSO could reduce the chemotherapeutic efficacy of human EC cells. Accordingly, the co-occurrence of CDDP and DMSO in treatment regimens may mediate accidental chemotherapeutic resistance or aberrant differentiation in EC cell components, with consequent negative effects on pharmacological efficacies in the clinical management of NSGCTs.

In the present study, DMSO reduced the expression of stemness-related markers, such as SOX2, in the human EC cells, while also reducing DNMT3L, which is a specific marker for human EC cells. DMSO is known to induce differentiation in ES cell lines $(25,26)$. Together with the perturbed differentiation of the human EC cells observed in response to DMSO within the present study, the findings also support a role for DMSO in modifying the stemness characteristics of EC cells and in mediating differentiation from pure EC cells to the other phenotypic components, including teratoma, choriocarcinoma and yolk sac tumors. Due to the acquisition of resistance to chemotherapy in parallel with aberrant differentiation $(23,24)$, the use of DMSO may be problematic when formulating drugs used to treat human EC cells $(23,24)$.

To evaluate whether the genomic demethylated status of EC cells could be affected by DMSO, the present study conducted COBRA for LINE1 repeats. This showed that DMSO increased the level of genomic DNA methylation in the human EC cells. These findings also suggested that the aberrant differentiation triggered by DMSO in the EC cells may be due, at least in part, to increased genomic methylation. Indeed, responsiveness to chemotherapy in TGCTs has been associated with a strong tendency to maintain the entire genome in a demethylated state (27). The lack of induction of the major drug efflux pumps in the study is supportive of a possible association between DNA methylation and the chemotherapeutic resistance phenomena observed in DMSO-exposed EC cells. Reduction of DNMT-3A and -3L expression following DMSO treatment in EC cells was correlated with the increased methylation of LINE1 elements; this is in agreement with a previous study that inhibited DNMT3L (28). In germ cells, DNMT3L expression occurs during a short perinatal period within non-dividing precursors of spermatogonial stem cells. The expression of DNMT3L declines rapidly after birth, together with reduction of DNMT-3A and -3B expression; in addition, retrotransposons undergo de novo methylation when the majority of prospermatogonia have differentiated into dividing spermatogonial stem cells (29). Taken together, the reduction in DNMT-3A and -3L expression is consistent with the increased methylation of LINE1 elements following treatment of human EC cells with DMSO.

In conclusion, the present study showed that DMSO can induce CDDP resistance in human EC cells, accompanied by perturbed differentiation and increased genomic methylation. DMSO could reduce chemotherapeutic efficacy in human EC cells, such that care is warranted with regard to its use during the clinical management of NSGCTs.

\section{Acknowledgements}

This study was supported by KAKENHI (Grant-in-Aids for Scientific Research) from the Ministry of Education, Culture, Sports, Science and Technology, Japan (grant no. 25293130).

\section{References}

1. Santos NC, Figueira-Coelho J, Martins-Silva J and Saldanha C: Multidisciplinary utilization of dimethyl sulfoxide: Pharmacological, cellular and molecular aspects. Biochem Pharmacol 65: 1035-1041, 2003.

2. Pectasides D, Pectasides E, Constantinidou A and Aravantinos G: Current management of stage I testicular non-seminomatous germ cell tumours. Crit Rev Oncol Hematol 70: 114-123, 2009.

3. Skakkebaek N: Possible carcinoma-in-situ of the testis. Lancet 2: 516-517, 1972.

4. Ulbright TM: Germ cell neoplasms of the testis. Am J Surg Pathol 17: 1075-1091, 1993.

5. Masters JR and Köberle B: Curing metastatic cancer: Lessons from testicular germ-cell tumours. Nat Rev Cancer 3: 517-525, 2003.

6. Sonneveld DJ, Hoekstra HJ, van der Graaf WT, et al: Improved long term survival of patients with metastatic nonseminomatous testicular germ cell carcinoma in relation to prognostic classification systems during the cisplatin era. Cancer 91: 1304-1315, 2001.

7. Freedman LS, Parkinson MC, Jones WG, et al: Histopathology in the prediction of relapse of patients with stage I testicular teratoma treated by orchidectomy alone. Lancet 2: 294-298, 1987.

8. Read G, Stenning SP, Cullen MH, et al: Medical Research Council prospective study of surveillance for stage I testicular teratoma. Medical Research Council Testicular Tumors Working Party. J Clin Oncol 10: 1762-1768, 1992.

9. Oliver RT, Ong J, Shamash J, et al: Long-term follow-up of Anglian Germ Cell Cancer Group surveillance versus patients with stage 1 nonseminoma treated with adjuvant chemotherapy. Urology 63: 556-561, 2004.

10. Cullen MH, Stenning SP, Parkinson MC, et al: Short-course adjuvant chemotherapy in high-risk stage I nonseminomatous germ cell tumors of the testis: A Medical Research Council report. J Clin Oncol 14: 1106-1113, 1996.

11. Guney S, Guney N, Sonmez NC and Ergenekon E: Risk-adapted management for patients with clinical stage I non-seminomatous germ cell tumour of the testis. Med Oncol 26: 136-142, 2009.

12. Vergouwe Y, Steyerberg EW, Eijkemans MJ, et al: Predictors of occult metastasis in clinical stage I nonseminoma: A systematic review. J Clin Oncol 21: 4092-4099, 2003.

13. Amato RJ, Ro JY, Ayala AG and Swanson DA: Risk-adapted treatment for patients with clinical stage I nonseminomatous germ cell tumor of the testis. Urology 63: 144-149, 2004.

14. Dunn TA, Schmoll HJ, Grünwald V, Bokemeyer C and Casper J: Comparative cytotoxicity of oxaliplatin and cisplatin in non-seminomatous germ cell cancer cell lines. Invest New Drugs 15: 109-114, 1997. 
15. Looijenga LH, Gillis AJ, Stoop HJ, Hersmus R and Oosterhuis JW: Chromosomes and expression in human testicular germ-cell tumors: Insight into their cell of origin and pathogenesis. Ann NY Acad Sci 1120: 187-214, 2007

16. Chano T, Ikegawa S, Kontani K, Okabe H, Baldini N and Saeki Y: Identification of RB1CC1, a novel human gene that can induce RB1 in various human cells. Oncogene 21: 1295-1298, 2002.

17. Minami K, Chano T, Kawakami T, Ushida H, Kushima R, Okabe H, Okada Y and Okamoto K: DNMT3L is a novel marker and is essential for the growth of human embryonal carcinoma. Clin Cancer Res 16: 2751-2759, 2010

18. Clark SJ, Harrison J, Paul CL and Frommer M: High sensitivity mapping of methylated cytosines. Nucleic Acids Res 22: 2990-2997, 1994.

19. Frevel MA, Sowerby SJ, Petersen GB and Reeve AE: Methylation sequencing analysis refines the region of $\mathrm{H} 19$ epimutation in Wilms tumor. J Biol Chem 274: 29331-29340, 1999.

20. Yang AS, Estécio MR, Doshi K, Kondo Y, Tajara EH and Issa JP. A simple method for estimating global DNA methylation using bisulfite PCR of repetitive DNA elements. Nucleic Acids Res 32: e38, 2004.

21. Ushida H, Chano T, Minami K, Kita H, Kawakami T, Okabe H, Okada Y and Okamoto K: Therapeutic potential of SOX2 inhibition for embryonal carcinoma. J Urol 187: 1876-1881. 2012.

22. Wohrle D, Salat U, Hameister H, Vogel W and Steinbach P: Demethylation, reactivation and destabilization of human fragile $\mathrm{X}$ full-mutation alleles in mouse embryocarcinoma cells. Am J Hum Genet 69: 504-515, 2001.
23. Wermann H, Stoop H, Gillis AJ, Honecker F, van Gurp RJ, Ammerpohl O, Richter J, Oosterhuis JW, Bokemeyer C and Looijenga LH: Global DNA methylation in fetal human germ cells and germ cell tumours: Association with differentiation and cisplatin resistance. J Pathol 221: 433-442. 2010.

24. Koul S, McKiernan JM, Narayan G, Houldsworth J, Bacik J, Dobrzynski DL, Assaad AM, Mansukhani M, Reuter VE, Bosl GJ, Chaganti RS and Murty VV: Role of promoter hypermethylation in cisplatin treatment response of male germ cell tumors. Mol Cancer 3: 16, 2004.

25. Adler S, Pellizzer C, Paparella M, Hartung T and Bremer S: The effects of solvents on embryonic stem cell differentiation. Toxicol In Vitro 20: 265-271, 2006.

26. Pal R, Mamidi MK, Das AK and Bhonde R: Diverse effects of dimethyl sulfoxide (DMSO) on the differentiation potential of human embryonic stem cells. Arch Toxicol 86: 651-661, 2012.

27. Okamoto K: Epigenetics: A way to understand the origin and biology of testicular germ cell tumors. Int J Urol 19: 504-511. 2012.

28. Ushida H, Kawakami T, Minami K, Chano T, Okabe H, Okada $\mathrm{Y}$ and Okamoto $\mathrm{K}$ : Methylation profile of DNA repetitive elements in human testicular germ cell tumor. Mol Carcinog 51: 711-722, 2012.

29. Bourc'his D and Bestor TH: Meiotic catastrophe and retrotransposon reactivation in male germ cells lacking Dnmt3L. Nature 431: 96-99, 2004. 